Supplement of Biogeosciences, 15, 4193-4203, 2018

https://doi.org/10.5194/bg-15-4193-2018-supplement

(C) Author(s) 2018. This work is distributed under

the Creative Commons Attribution 4.0 License.

(c) (1)

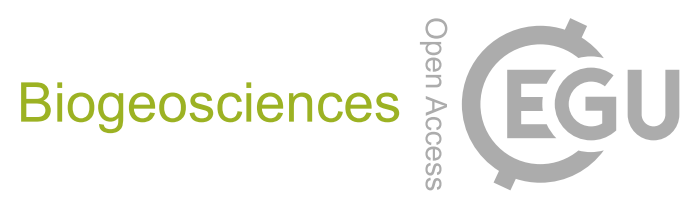

Supplement of

\title{
The strategies of water-carbon regulation of plants in a subtropical primary forest on karst soils in China
}

\author{
Jing Wang et al. \\ Correspondence to: Xuefa Wen (wenxf@igsnrr.ac.cn) and Xinyu Zhang (zhangxy@igsnrr.ac.cn)
}

The copyright of individual parts of the supplement might differ from the CC BY 4.0 License. 

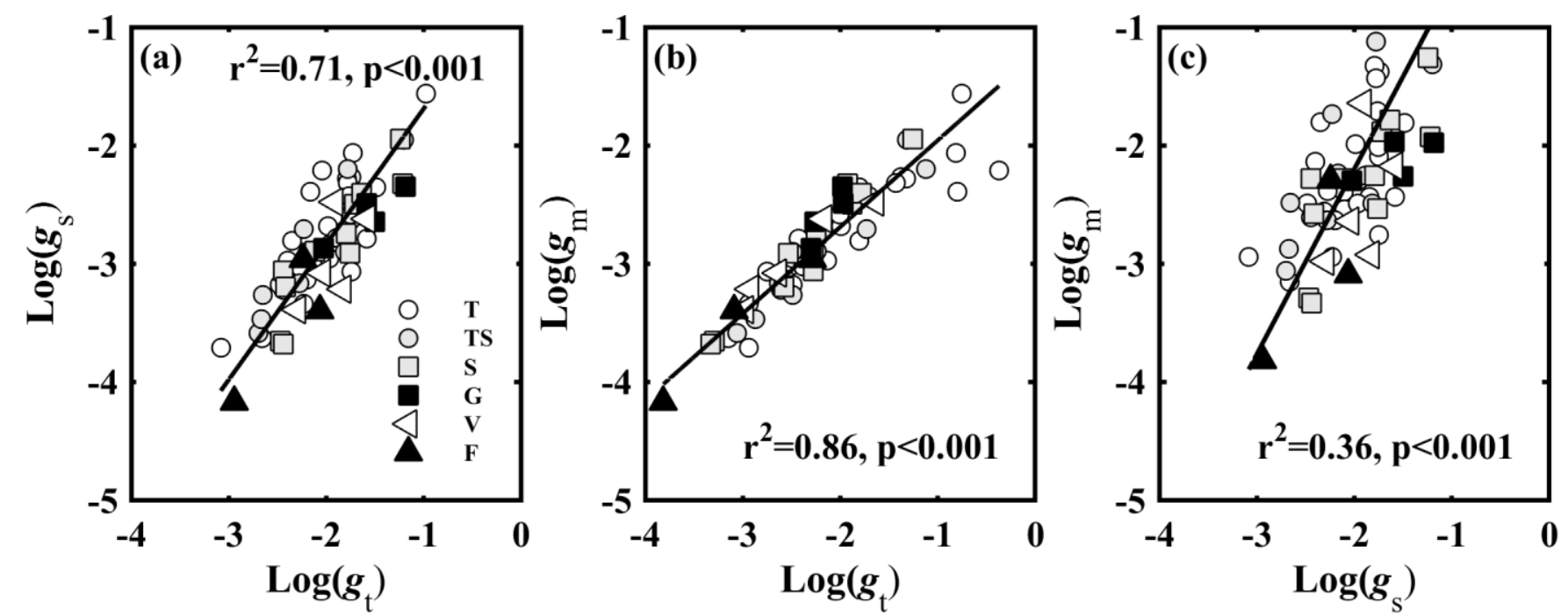

Figure S1 Relationship between (a) stomatal conductance to $\mathrm{CO}_{2}\left(g_{\mathrm{s}}, \mathrm{mol} \mathrm{CO}_{2} \mathrm{~m}^{-2} \mathrm{~s}^{-1}\right)$ and total conductance to $\mathrm{CO}_{2}\left(g_{\mathrm{t}}, \mathrm{mol} \mathrm{CO}_{2} \mathrm{~m}^{-2} \mathrm{~s}^{-1}\right)$; (b) mesophyll conductance to $\mathrm{CO}_{2}\left(g_{\mathrm{m}}, \mathrm{mol} \mathrm{CO}_{2} \mathrm{~m}^{-2} \mathrm{~s}^{-1}\right)$ and gt; and (c) $g_{\mathrm{s}}$ and $g_{\mathrm{m}}$. Lines refer to regression line for 63 species. T, TS, $\mathrm{S}, \mathrm{G}, \mathrm{V}$, and $\mathrm{F}$ represent Tree, Tree/Shrub, Shrub, Grass, Vine, and Fern, respectively. 


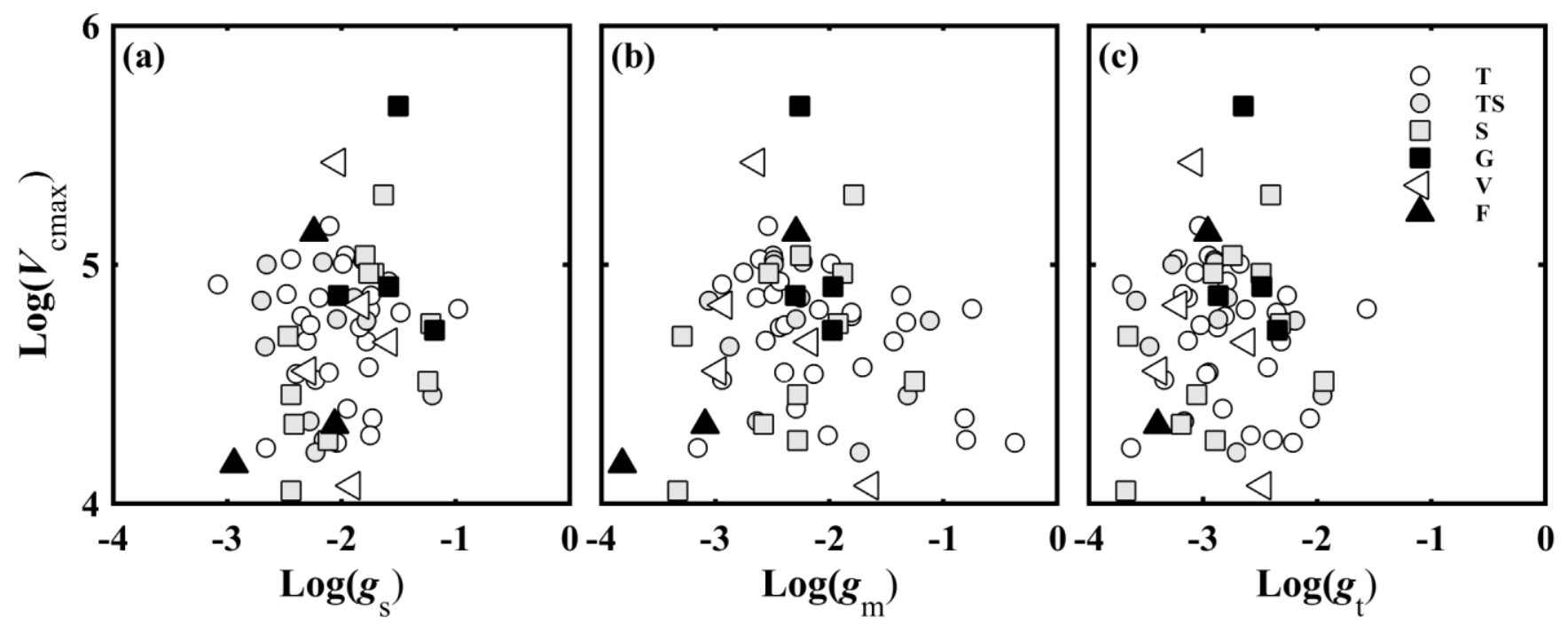

Figure S2 Relationship between (a) stomatal conductance to $\mathrm{CO}_{2}\left(g_{\mathrm{s}}, \operatorname{mol~CO}_{2} \mathrm{~m}^{-2} \mathrm{~s}^{-1}\right)$ and the maximum carboxylase activity of Rubisco $\left(V_{\mathrm{cmax}}\right.$, $\left.\mu \mathrm{mol} \mathrm{CO} \mathrm{CO}^{-2} \mathrm{~s}^{-1}\right)$; (b) mesophyll conductance to $\mathrm{CO}_{2}\left(g_{\mathrm{m}}, \mathrm{mol} \mathrm{CO} \mathrm{C}^{-2} \mathrm{~s}^{-1}\right)$ and $V_{\text {cmax }}$; and (c) total conductance to $\mathrm{CO}_{2}\left(g_{\mathrm{t}}\right)$ and $V_{\mathrm{cmax}}$. Lines refer to regression line for 63 species. T, TS, S, G, V, and F represent Tree, Tree/Shrub, Shrub, Grass, Vine, and Fern, respectively. 


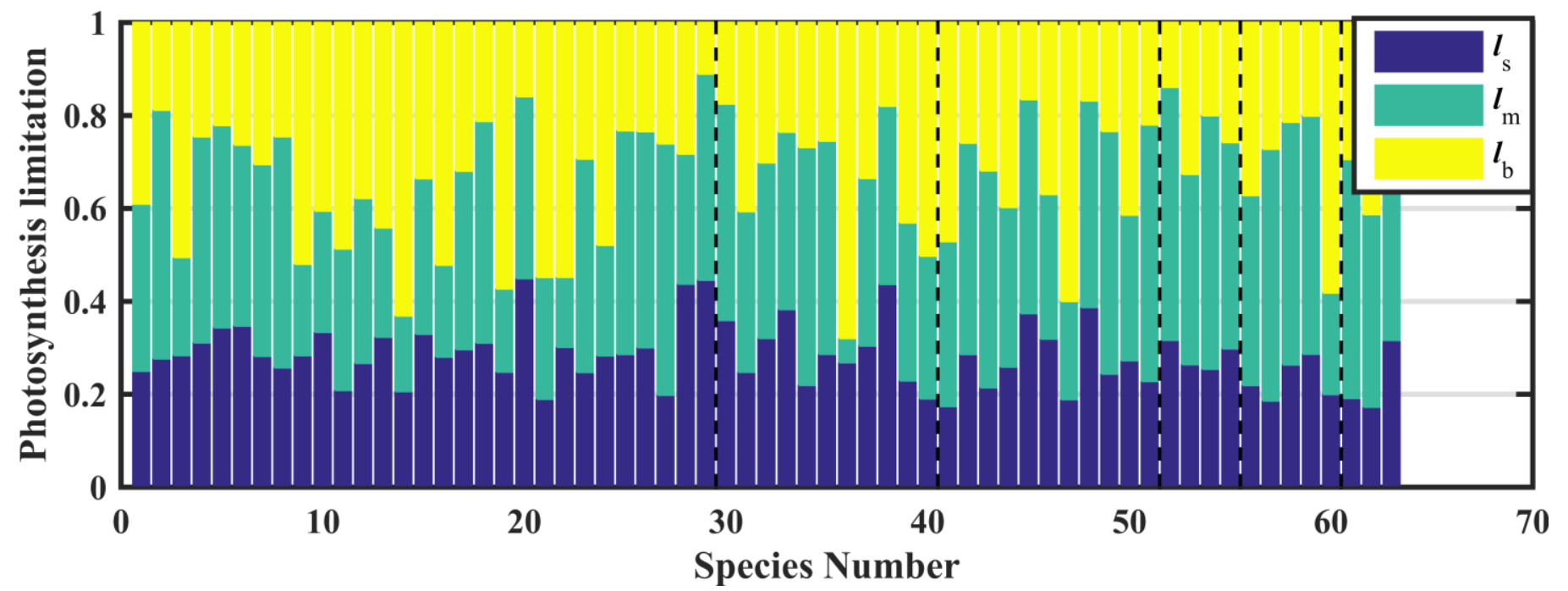

Figure S3 The limitation of (a) stomatal conductance to $\mathrm{CO}_{2}\left(g_{\mathrm{s}}\right)$ on photosynthesis rate $(\mathrm{A})\left(l_{\mathrm{s}}\right)$, (b) mesophyll conductance to $\mathrm{CO}_{2}\left(g_{\mathrm{m}}\right)$ on $A\left(l_{\mathrm{m}}\right)$ and (c) the maximum carboxylase activity of Rubisco $\left(V_{\mathrm{cmax}}\right)$ on $\mathrm{A}\left(l_{\mathrm{b}}\right)$. 


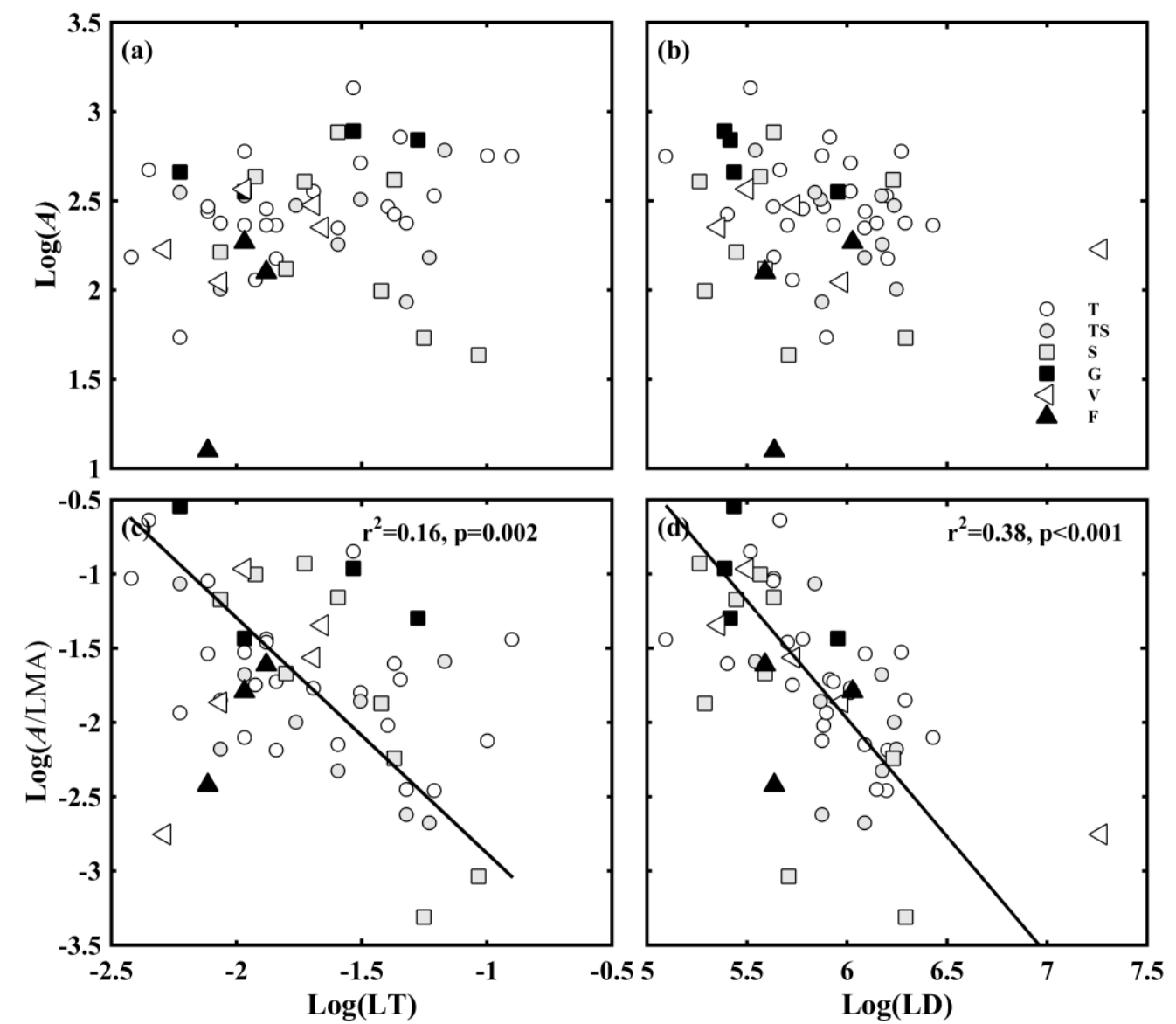

Figure S4 Relationship between (a) light-saturated net photosynthesis (A) and the leaf thickness (LT); (b) $A$ and he leaf density (LD); (c) the ratio of $A$ to leaf mass per area (LMA) (A/LMA); and (d) A/LMA and LD. Lines refer to regression line for 63 species. T, TS, S, G, V, and F represent Tree, Tree/Shrub, Shrub, Grass, Vine, and Fern, respectively. 

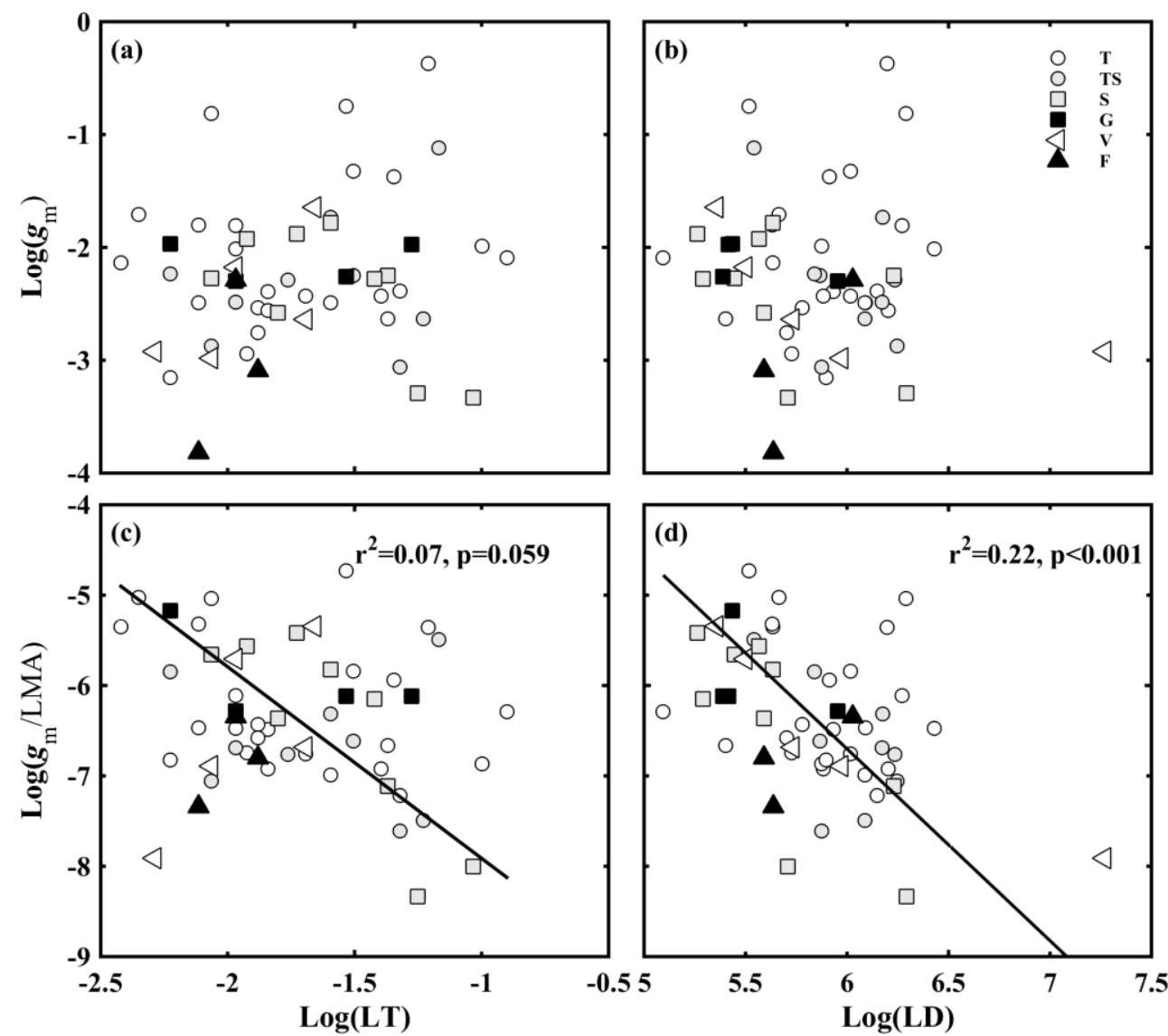

Figure S5 Relationship between (a) the mesophyll conductance to $\mathrm{CO}_{2}\left(g_{\mathrm{m}}\right)$ and the leaf thickness (LT); (b) $g_{\mathrm{m}}$ and he leaf density (LD); (c) the ratio of $g_{\mathrm{m}}$ to leaf mass per area (LMA) ( $\left.g_{\mathrm{m}} / \mathrm{LMA}\right)$; and (d) $g_{\mathrm{m}} / \mathrm{LMA}$ and LD. Lines refer to regression line for 63 species. T, TS, S, G, V, and F represent Tree, Tree/Shrub, Shrub, Grass, Vine, and Fern, respectively. 

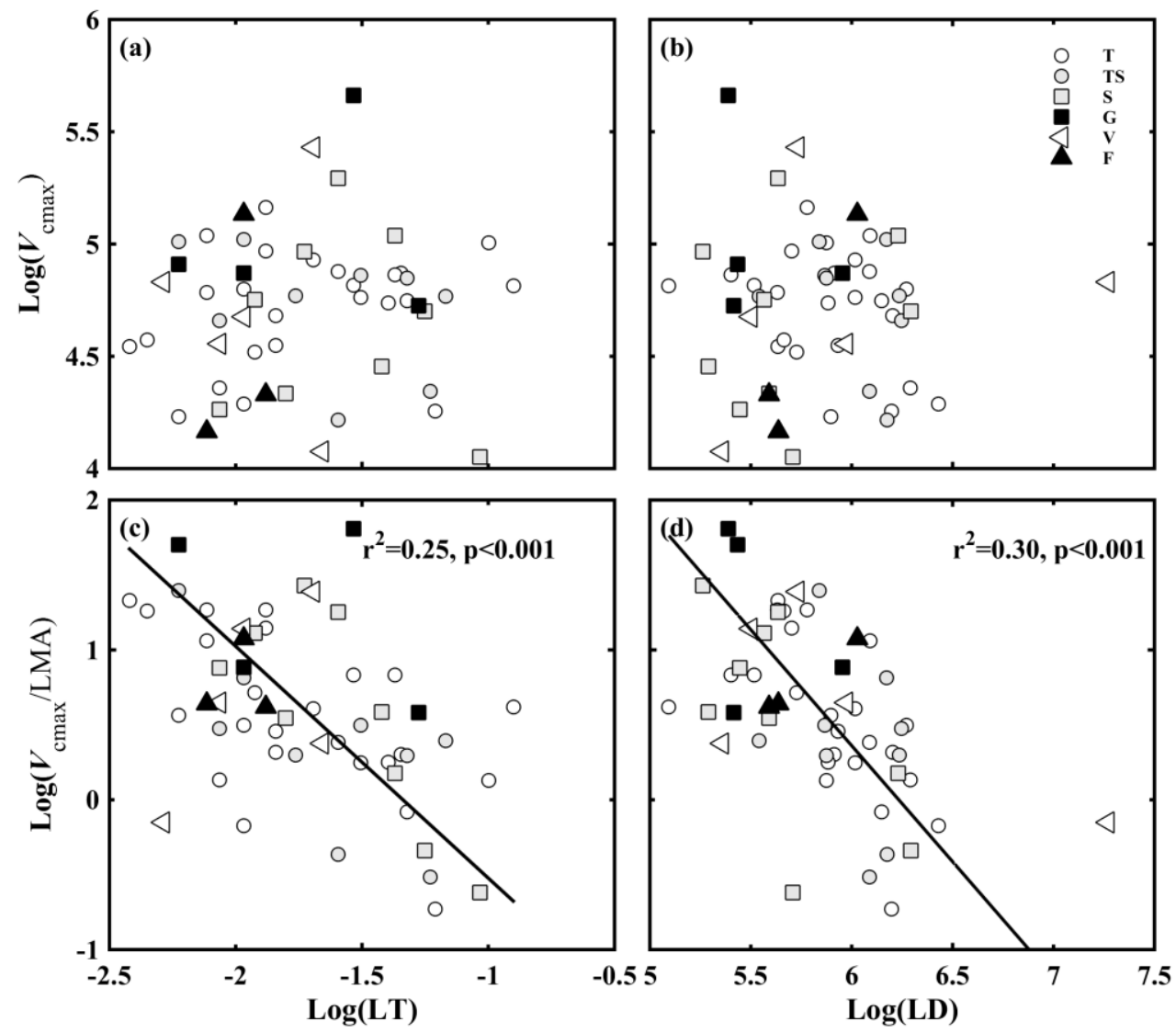

Figure S6 Relationship between (a) the maximum carboxylase activity of Rubisco $\left(V_{\text {cmax }}\right)$ and the leaf thickness (LT); (b) $V_{\text {cmax }}$ and he leaf density (LD); (c) the ratio of $V_{\text {cmax }}$ to leaf mass per area (LMA) ( $V_{\text {cmax }} / \mathrm{LMA}$ ); and (d) $V_{\text {cmax }} / \mathrm{LMA}$ and LD. Lines refer to regression line for 63 species. T, TS, S, G, V, and F represent Tree, Tree/Shrub, Shrub, Grass, Vine, and Fern, respectively. 


\section{Tables}

Table S1 Details information about the 63 species in the subtropical primary forest in Southwest China.

\begin{tabular}{llll}
\hline Species & Plant family & Life form \\
\hline Broussonetia papyifera (Linn.) L'Hert. ex Vent. & Moraceae & Tree & Deciduous Woody \\
Machilus microcarpa Hemsl. & Lauraceae & Tree & Evergreen Woody \\
Melia azedarach L. & Meliaceae & Tree & Deciduous Woody \\
Populus x canadensis Moench. & Salicaceae & Tree & Deciduous Woody \\
Camptotheca acuminata Decne. & Nyssaceae & Tree & Deciduous Woody \\
Cinnamomum bodinieri Levl. & Lauraceae & Tree & Evergreen Woody \\
Catalpa ovata G. Don & Bignoniaceae & Tree & Deciduous Woody \\
Toona sinensis (A. Juss.) Roem. & Meliaceae & Tree & Deciduous Woody \\
Sapium sebiferum (Linn.) Roxb. & Euphorbiaceae & Tree & Deciduous Woody \\
Cladrastis platycarpa (Maxim.) Makino & Leguminosae & Tree & Deciduous Woody \\
Ulmus pumila L. & Ulmaceae & Tree & Deciduous Woody \\
Ilex macrocarpa Oliv. & Aquifoliaceae & Tree & Deciduous Woody \\
Vitex canescens Kurz & Verbenaceae & Tree & Deciduous Woody \\
Eriobotrya japonica (Thunb.) Lindl. & Rosaceae & Tree & Evergreen Woody \\
Morus alba L. & Moraceae & Tree & Deciduous Woody \\
Prunus salicina Lindl. & Rosaceae & Tree & Deciduous Woody \\
Eucommia ulmoides Oliver & Eucommiaceae & Tree & Deciduous Woody \\
Platycarya strobilacea Sieb. et Zucc. & Juglandaceae & Tree & Deciduous Woody \\
Kalopanax septemlobus (Thunb.) Koidz. & Araliaceae & Tree & Deciduous Woody \\
Zanthoxylum armatum DC. & Rutaceae & Tree & Deciduous Woody \\
Pyrus calleryana & Rosaceae & Tree & Deciduous Woody \\
Amygdalus persica L. var. & Rosaceae & Tree & Deciduous Woody \\
\hline
\end{tabular}




\begin{tabular}{llll}
\hline Euonymus meaackii Rupr. & Celastraceae & Tree & Deciduous Woody \\
Zanthoxylum ovalifolium Wight & Rutaceae & Tree & Deciduous Woody \\
Cerasus scopulorum (Koehne) Yu et Li & Rosaceae & Tree & Deciduous Woody \\
Carpinus pubescens Burk. & Betulaceae & Tree & Deciduous Woody \\
Lithocarpus confinis Huang & Fagaceae & Tree & Evergreen Woody \\
Celtis sinensis Pers. & Ulmaceae & Tree & Deciduous Woody \\
Diospyros kaki Thunb. var. silvestris Makino & Ebenaceae & Tree & Deciduous Woody \\
Ligustrum lucidum Ait. & Oleaceae & Tree/Shrub Deciduous Woody \\
Rhamnus leptophylla Schneid. & Rhamnaceae & Tree/Shrub Deciduous Woody \\
Lindera communis Hemsl. & Lauraceae & Tree/Shrub Evergreen Woody \\
Itea yunnanensis Franch & Saxifragaceae & Tree/Shrub Evergreen Woody \\
Pittosporum brevicalyx (Oliv.) Gagnep & Pittosporaceae & Tree/Shrub Evergreen Woody \\
Litsea rubescens Lec. & Lauraceae & Tree/Shrub Deciduous Woody \\
Rhus chinensis Mill. & Anacardiaceae & Tree/Shrub Deciduous Woody \\
Alangium chinense (Lour.) Harms & Alangiaceae & Tree/Shrub Deciduous Woody \\
Evodia rutaecarpa (Juss.) Benth. & Rutaceae & Tree/Shrub Deciduous Woody \\
Machilus cavaleriei Levl. & Lauraceae & Tree/Shrub Evergreen Woody \\
Debregeasia longifolia (Burm. f.) Wedd. & Urticaceae & Tree/Shrub Deciduous Woody \\
Ziziphus jujuba Mill. var. spinosa (Bunge) Hu ex H. F. Chow & Rhamnaceae & Shrub & Deciduous Woody \\
Rubus inopertus (Diels) Focke & Rosaceae & Shrub & Deciduous Woody \\
Coriaria nepalensis Wall. & Coriariaceae & Shrub & Deciduous Woody \\
Celastrus orbiculatus Thunb. & Celastraceae & Shrub Deciduous Woody \\
Wikstroemia scytophylla Diels & Thymelaeaceae & Shrub & Deciduous Woody \\
Viburnum foetidum Wall. var. ceanothoides (C. H. Wright) Hand.-Mazz. Caprifoliaceae & Shrub & Deciduous Woody \\
Hedera nepalensis K. Koch var. sinensis (Tobl.) Rehd. & Araliaceae & Shrub & Deciduous Woody \\
Rubus parvifolius L. & Rosaceae & Shrub & Deciduous Woody \\
\hline
\end{tabular}




\begin{tabular}{|c|c|c|c|c|}
\hline Rosa roxbunghii & Rosaceae & Shrub & Deciduous & Woody \\
\hline Mallotus repandus (Willd.) Muell. Arg. & Euphorbiaceae & Shrub & Deciduous & Woody \\
\hline Mahonia bealei (Fort.) Carr. & Berberidaceae & Shrub & Evergreen & Woody \\
\hline Fallopia multiflora (Thunb.) Harald. & Polygonaceae & Grass & & Herb \\
\hline Conyza canadensis (L.) Cronq. & Compositae & Grass & & Herb \\
\hline Ipomoea batatas (L.) Lam. & Convolvulaceae & Grass & & Herb \\
\hline Senecio scandens Buch.-Ham. ex D. Don & Compositae & Grass & & Herb \\
\hline Vitis piasezkii Maxim. & Vitaceae & Vien & Deciduous & Woody \\
\hline Clematis urophylla Franch. & Ranunculaceae & Vien & Deciduous & Woody \\
\hline Bauhinia glauca (Wall. ex Benth.) Benth. & Leguminosae & Vien & Evergreen & Woody \\
\hline Caesalpinia decapetala (Roth) Alston & Leguminosae & Vien & Deciduous & Woody \\
\hline Paederia scandens (Lour.) Merr. & Rubiaceae & Vien & & Herb \\
\hline Cyclosorus parasiticus (L.) Farwell. & Thelypteridaceae & Fern & & \\
\hline Cyrtomium fortunei J. Sm. & Dryopteridaceae & Fern & & \\
\hline Pteris vittata L. & Pteridaceae & Fern & & \\
\hline
\end{tabular}


Table S2 Coefficients of determination of linear regressions of fig. 1-4 and fig.6-7.

\begin{tabular}{|c|c|c|c|c|c|c|c|c|c|c|c|c|c|}
\hline & & Fig.1 & & Fig. 2 & & Fig. 3 & & Fig.4 & & Fig.6 & & Fig. 7 & \\
\hline Subgraph & Life form & $\mathrm{R}^{2}$ & $\mathrm{P}$ & $\mathrm{R}^{2}$ & $\mathrm{P}$ & $\mathrm{R}^{2}$ & $\mathrm{P}$ & $\mathrm{R}^{2}$ & $\mathrm{P}$ & $\mathrm{R}^{2}$ & $\mathrm{P}$ & $\mathrm{R}^{2}$ & $\mathrm{P}$ \\
\hline \multirow{4}{*}{$\mathrm{a}$} & Total & 0.35 & 0.000 & 0.09 & 0.018 & 0.67 & 0.000 & 0.19 & 0.000 & 0.00 & 0.922 & 0.20 & 0.000 \\
\hline & Tree & 0.49 & 0.000 & 0.14 & 0.048 & 0.67 & 0.000 & 0.42 & 0.000 & 0.03 & 0.401 & 0.11 & 0.083 \\
\hline & $\begin{array}{l}\text { Tree/Shru } \\
\text { b }\end{array}$ & 0.70 & 0.001 & 0.49 & 0.016 & 0.79 & 0.000 & 0.57 & 0.007 & 0.24 & 0.126 & 0.07 & 0.438 \\
\hline & Shrub & 0.29 & 0.085 & 0.10 & 0.350 & 0.78 & 0.000 & 0.11 & 0.314 & 0.00 & 1.000 & 0.20 & 0.173 \\
\hline \multirow[t]{2}{*}{ b } & $\begin{array}{l}\text { Tree/Shru } \\
\text { b }\end{array}$ & 0.84 & 0.000 & 0.67 & 0.002 & 0.68 & 0.002 & 0.78 & 0.000 & 0.70 & 0.001 & 0.78 & 0.000 \\
\hline & Shrub & 0.60 & 0.005 & 0.50 & 0.015 & 0.75 & 0.001 & 0.42 & 0.031 & 0.22 & 0.142 & 0.56 & 0.008 \\
\hline \multirow{2}{*}{$\mathrm{c}$} & Total & 0.55 & 0.000 & 0.59 & 0.000 & 0.76 & 0.000 & 0.38 & 0.000 & 0.00 & 0.934 & & \\
\hline & Tree & 0.68 & 0.000 & 0.67 & 0.000 & 0.70 & 0.000 & 0.63 & 0.000 & 0.01 & 0.549 & & \\
\hline \multirow{3}{*}{ d } & Tree & 0.36 & 0.001 & & & 0.09 & 0.121 & 0.34 & 0.001 & 0.08 & 0.133 & & \\
\hline & $\begin{array}{l}\text { Tree/Shru } \\
\text { b }\end{array}$ & 0.40 & 0.038 & & & 0.02 & 0.714 & 0.52 & 0.013 & 0.19 & 0.180 & & \\
\hline & Shrub & 0.04 & 0.552 & & & 0.53 & 0.011 & 0.01 & 0.734 & 0.06 & 0.471 & & \\
\hline
\end{tabular}

